\title{
Vizing-like conjecture for the upper domination of Cartesian products of graphs - the proof
}

\author{
Boštjan Brešar* \\ University of Maribor, FEECS \\ Smetanova 17, 2000 Maribor, Slovenia \\ bostjan.bresar@uni-mb.si \\ Submitted: Jul 7, 2004; Accepted: Jun 30, 2005; Published: Jul 19, 2005 \\ Mathematics Subject Classifications: 05C69, 05C99
}

\begin{abstract}
In this note we prove the following conjecture of Nowakowski and Rall: For arbitrary graphs $G$ and $H$ the upper domination number of the Cartesian product $G \square H$ is at least the product of their upper domination numbers, in symbols: $\Gamma(G \square H) \geq \Gamma(G) \Gamma(H)$.
\end{abstract}

A conjecture posed by Vizing [7] in 1968 claims that

Vizing's conjecture: For any graphs $G$ and $H, \gamma(G \square H) \geq \gamma(G) \gamma(H)$,

where $\gamma$, as usual, denotes the domination number of a graph, and $G \square H$ is the Cartesian product of graphs $G$ and $H$. It became one of the main problems of graph domination, cf. surveys [2] and [4, Section 8.6], and two recent papers $[1,6]$.

The unability of proving or disproving it lead authors to pose different variations of the original problem. Several such variations were studied by Nowakowski and Rall in the paper [5] from 1996. In particular, they proposed the following

Conjecture (Nowakowski, Rall): For any graphs $G$ and $H, \Gamma(G \square H) \geq \Gamma(G) \Gamma(H)$,

where $\Gamma$ denotes the upper domination of a graph. In this note we prove this conjecture. In fact, if both graphs $G$ and $H$ are nontrivial (i.e. have at least two vertices) we prove the following slightly stronger bound:

$$
\Gamma(G \square H) \geq \Gamma(G) \Gamma(H)+1 .
$$

\footnotetext{
*Supported by the Ministry of Education, Science and Sport of Slovenia under the grant Z1-30730101-01.
} 
We start with basic definitions. For graphs $G$ and $H$, the Cartesian product $G \square H$ is the graph with vertex set $V(G) \times V(H)$ where two vertices $\left(u_{1}, v_{1}\right)$ and $\left(u_{2}, v_{2}\right)$ are adjacent if and only if either $u_{1}=u_{2}$ and $v_{1} v_{2} \in E(H)$ or $v_{1}=v_{2}$ and $u_{1} u_{2} \in E(G)$. For a set of vertices $S \subseteq V(G) \times V(H)$ let $p_{G}(S), p_{H}(S)$ denote the natural projections of $S$ to $V(G)$ and $V(H)$, respectively.

A set $S \subset V(G)$ of vertices in a graph $G$ is called dominating if for every vertex $v \in$ $V(G) \backslash S$ there exists a vertex $u \in S$ that is adjacent to $v$. A dominating set $S$ is minimal dominating set if no proper subset of $S$ is dominating. Minimal dominating sets give rise to our central definition. The upper domination number $\Gamma(G)$ of a graph $G$ is the maximum cardinality of a minimal dominating set in $G$. Recall that the domination number $\gamma(G)$ is the minimum cardinality of a (minimal) dominating set in $G$. The following fundamental result due to Ore, cf. [3, Theorem 1.1], characterizes minimal dominating sets in graphs.

Theorem $1 A$ dominating set $S$ is a minimal dominating set if and only if for every vertex $u \in S$ one of the following two conditions holds:

(i) $u$ is not adjacent to any vertex of $S$,

(ii) there exists a vertex $v \in V(G) \backslash S$ such that $u$ is the only neighbor of $v$ from $S$.

Based on Ore's theorem we present a partition of the vertex set of a graph depending on a given minimal dominating set. Let $D_{G}$ be a minimal dominating set of a graph $G$. If for a vertex $u \in D_{G}$ the condition (ii) of Theorem 1 holds, then we say that $v$ is a private neighbor of $u$ (that is, $v$ is adjacent only to $u$ among vertices of $D_{G}$ ). Note that $u$ can have more than one private neighbor. Also note that for a vertex $u$ of $D_{G}$ both conditions of Theorem 1 can hold at the same time, that is, it can have a private neighbor and be nonadjacent to all other vertices of $D_{G}$. Denote by $D_{G}^{\prime}$ the set vertices of $D_{G}$ that have a private neighbor, and by $P_{G}$ the set of vertices of $V(G) \backslash D_{G}$ which are private neighbors of some vertex of $D_{G}^{\prime}$. By $N_{G}$ we denote the set of vertices of $V(G) \backslash D_{G}$ which are adjacent to a vertex of $D_{G}^{\prime}$ but are not private neighbors of any vertex of $D_{G}^{\prime}$. Set $D_{G}^{\prime \prime}=D_{G} \backslash D_{G}^{\prime}$ denoting the vertices of $D_{G}$ which do not have private neighbors (so they must enjoy condition (i) of the theorem), and finally let the remaining set be $R_{G}$, that is $R_{G}=V(G) \backslash\left(D_{G} \cup P_{G} \cup N_{G}\right)$. We will skip the indices if the graph $G$ will be understood from the context. Note that given a minimal dominating set $D$ of a graph $G$, the sets $D^{\prime}, D^{\prime \prime}, P, N$ and $R$ form a partition of the vertex set $V(G)$. In addition, some pairs of sets must clearly have adjacent vertices (like $D^{\prime}$ and $P$ ), while some other pairs of sets clearly do not have any adjacent vertices (like $D^{\prime}$ and $D^{\prime \prime}$ ). The situation is presented in Figure 1, where doubled line indicates that between two sets there must be edges, a normal line indicates that between the two sets edges are possible (but are not necessary), and no line between two sets means no edges are possible. Note that every vertex of $R$ is adjacent to a vertex of $D^{\prime \prime}$, and that every vertex of $N \cup P$ is adjacent to a vertex of $D^{\prime}$. Of course, some of the sets could also be empty for some dominating sets.

If $A$ and $B$ are two subsets of the vertex set of a graph we say that $A$ dominates (vertices of) $B$ if every vertex of $B$ has a neighbor in $A$ or is a vertex of $A$. We may then also say that $B$ is dominated by (vertices of) $A$. 


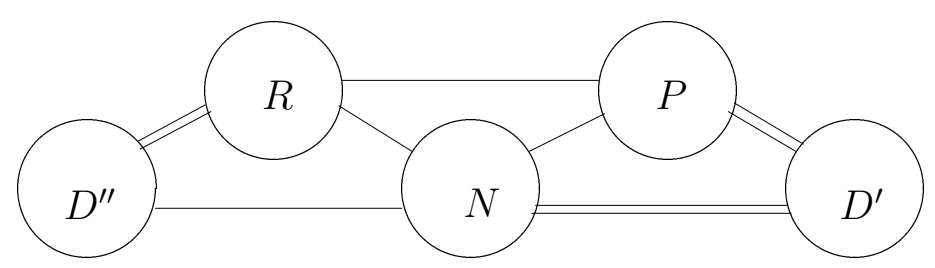

Figure 1: Partition of the vertex set

In the proof of the conjecture we will use two special sets, obtained by an operation of completion of a certain set to a set that dominates a specified set of vertices of a graph. Let us present these operations.

1. Let $G$ be a graph, $D$ a minimal dominating set, and $D^{\prime}, D^{\prime \prime}, P, N, R$ the corresponding sets that form a partition of $V(G)$. Let $I$ be a subset of $R$. By $S P\left(D^{\prime}, I\right)$ we denote a subset of vertices from $D^{\prime}$ such that $S P\left(D^{\prime}, I\right) \cup I$ dominates $P \cup N$ (it need not be a dominating set of entire graph), and is minimal in the folowing sense. For each vertex $u$ of $S P\left(D^{\prime}, I\right)$

$\left(^{*}\right)$ there exists a vertex $v \in P \cup N$ such that $u$ is its only neighbor from $S P\left(D^{\prime}, I\right) \cup I$.

That such a set always exists follows from two facts. First $D^{\prime}$ itself dominates $P \cup N$ (and if $I$ does not dominate any vertex of $P \cup N$, then $D^{\prime}$ is already minimal in the above sense). Now, minimality condition can be easily achieved by adding to $S P\left(D^{\prime}, I\right)$ vertex by vertex from $D^{\prime}$ that are needed to dominate vertices of $P$ (those which are not dominated by $I$ ), and after that, if some of the vertices of $N$ remain undominated, additional vertices from $D^{\prime}$ are added to $S P\left(D^{\prime}, I\right)$.

2. The second operation is a modification of the first, where we start with a subset of $D^{\prime \prime} \cup R$ instead of just $R$. So let $J$ be a subset of $D^{\prime \prime} \cup R$. By $S P^{\prime}\left(D^{\prime}, J\right)$ we denote a minimal set of vertices from $D^{\prime}$ such that $N \cup P$ is dominated by vertices of $J \cup S P^{\prime}\left(D^{\prime}, J\right)$.

Theorem 2 For any nontrivial graphs $G$ and $H$,

$$
\Gamma(G \square H) \geq \Gamma(G) \Gamma(H)+1 .
$$

Proof. For the proof we will construct a minimal dominating set $D$ of $G \square H$ having enough vertices. Let $D_{G}$ and $D_{H}$ be minimal dominating sets of $G$ and $H$, respectively, with maximum cardinality, that is $\left|D_{G}\right|=\Gamma(G)$ and $\left|D_{H}\right|=\Gamma(H)$.

Consider first the case where in one of the factors (say $G$ ) the set $D^{\prime \prime}$ is empty. Then $D:=D_{G}^{\prime} \times V(H)$ is clearly a minimal dominating set (every vertex of $D$ has a private neighbor) with more than $\Gamma(G) \Gamma(H)+1$ vertices. If both $D_{G}^{\prime}$ and $D_{H}^{\prime}$ are empty, then let $D:=\left(D_{G}^{\prime \prime} \times D_{H}^{\prime \prime}\right) \cup I$ where $I$ is a maximum independent set of the subgraph induced by $\left(V(G) \backslash D_{G}^{\prime \prime}\right) \times\left(V(H) \backslash D_{H}^{\prime \prime}\right)$. Since $I$ is obviously nonempty, $D$ is a minimal dominating set (it is a maximal independent set) with at least $\Gamma(G) \Gamma(H)+1$ vertices.

In the sequel we may assume without loss of generality that $D_{H}^{\prime} \neq \emptyset, D_{H}^{\prime \prime} \neq \emptyset$ and $D_{G}^{\prime \prime} \neq \emptyset$. We will construct $D$ as a union of six pairwise disjoint sets (in the case $D_{G}^{\prime}=\emptyset$ the last three sets will be empty). 
Let the first set be $D_{1}=D_{G}^{\prime \prime} \times D_{H}$ (note that it has $\left|D_{G}^{\prime \prime}\right| \cdot \Gamma(H)$ vertices). Let the second set $\left(D_{2}\right)$ be a maximum independent set $I$ of the subgraph induced by $R_{G} \times R_{H}$.

For each $x \in R_{G}$ denote by $I_{x}$ the set $I \cap(\{x\} \times V(H))$. Let $S P\left(D_{H}^{\prime}, p_{H}\left(I_{x}\right)\right)$ be the subset of $D_{H}^{\prime}$ obtained by the operation defined above, and consider the corresponding subset of $G \square H$, that is $\{x\} \times S P\left(D_{H}^{\prime}, p_{H}\left(I_{x}\right)\right)$. Let the third set of $D$ be the union of all such sets, that is

$$
D_{3}=\bigcup_{x \in R_{G}}\{x\} \times S P\left(D_{H}^{\prime}, p_{H}\left(I_{x}\right)\right)
$$

which is obviously a subset of $R_{G} \times D_{H}^{\prime}$.

The fourth set is obtained similarly by reversing the roles of $G$ and $H$. That is for each $y \in R_{H}$ denote by $I_{y}$ the set $I \cap(V(G) \times\{y\})$. Then $S P\left(D_{G}^{\prime}, p_{G}\left(I_{y}\right)\right)$ is a subset of $D_{G}^{\prime}$, and let

$$
D_{4}=\bigcup_{y \in R_{H}} S P\left(D^{\prime}, p_{G}\left(I_{y}\right)\right) \times\{y\}
$$

which is a subset of $D_{G}^{\prime} \times R_{H}$.

For each $y \in D_{H}^{\prime}$ let $J_{y}$ be the set of vertices from $V(G) \times\{y\}$ that are already included in $D$. That is

$$
J_{y}=\left(D_{1} \cup D_{3}\right) \cap(V(G) \times\{y\}),
$$

and for each such set add to $D$ vertices in $V(G) \times\{y\}$ by using the second operation from above:

$$
D_{5}=\bigcup_{y \in D_{H}^{\prime}} S P^{\prime}\left(D_{G}^{\prime}, p_{G}\left(J_{y}\right)\right) \times\{y\}
$$

which is clearly a subset of $D_{G}^{\prime} \times D_{H}^{\prime}$.

Finally, set

$$
D_{6}=D_{G}^{\prime} \times\left(V(H) \backslash\left(D_{H}^{\prime} \cup R_{H}\right)\right) .
$$

Since $\left|P_{H}\right| \geq\left|D_{H}^{\prime}\right|$, we infer $\left|V(H) \backslash\left(D_{H}^{\prime} \cup R_{H}\right)\right| \geq\left|D_{H}\right|$, and so $\left|D_{6}\right| \geq\left|D_{G}^{\prime}\right| \cdot \Gamma(H)$.

Now, as said before let $D=D_{1} \cup D_{2} \cup D_{3} \cup D_{4} \cup D_{5} \cup D_{6}$ and obviously the six sets are pairwise disjoint. From previous observations we get

$$
\left|D_{1}\right|+\left|D_{6}\right| \geq\left|D_{G}^{\prime \prime}\right| \cdot \Gamma(H)+\left|D_{G}^{\prime}\right| \cdot \Gamma(H)=\left|D_{G}\right| \cdot \Gamma(H)=\Gamma(G) \Gamma(H) .
$$

Since $D_{G}^{\prime \prime} \neq \emptyset$ and $D_{H}^{\prime \prime} \neq \emptyset$, we get $R_{G} \cup N_{G} \neq \emptyset$ and $R_{H} \cup N_{H} \neq \emptyset$. If $R_{G}=\emptyset$ then $D_{5}$ must be nonempty. If $R_{G} \neq \emptyset$ and $R_{H}=\emptyset$ then $D_{3}$ must be nonempty. Finally, $R_{G} \neq \emptyset$ and $R_{H} \neq \emptyset$ implies $D_{2}$ is nonempty. We infer that $|D| \geq \Gamma(G) \Gamma(H)+1$. (This is even easier to deduce if $D_{G}^{\prime}=\emptyset$.)

In the rest of the proof we (must) show that $D$ is a minimal dominating set of $G \square H$. To prove that $D$ is a dominating set we will partition $G \square H$ and check for each part that is dominated by $D$.

Vertices of $D_{G}^{\prime \prime} \times V(H)$ are obviously dominated by $D_{1}$.

Next consider vertices of $R_{G} \times V(H)$. Vertices of $R_{G} \times R_{H}$ are dominated by $I=D_{2}$, since it is its maximum (and thus maximal) independent set. Vertices of $R_{G} \times D_{H}$ are 
dominated by $D_{1}$, and other vertices of $R_{G} \times V(H)$ are dominated by $D_{2} \cup D_{3}$ (by using the operation $S P$ ).

Vertices of $D_{G}^{\prime} \times V(H)$ are dominated by $D_{6}$. Indeed, recall that $D_{6}$ is $D_{G}^{\prime} \times\left(P_{H} \cup\right.$ $D_{H}^{\prime \prime} \cup N_{H}$ ), and that $P_{H} \cup D_{H}^{\prime \prime} \cup N_{H}$ is a dominating set of $H$.

Vertices of $P_{G} \times V(H)$ and of $N_{G} \times V(H)$ are dominated as follows. If $y \in V(H)$ is a vertex of $R_{H}$ then $\left(P_{G} \cup N_{G}\right) \times\{y\}$ is dominated by $D_{2} \cup D_{4}$ by using operation $S P$. If $y$ is in $D_{H}^{\prime}$ then $\left(P_{G} \cup N_{G}\right) \times\{y\}$ is dominated by $D_{1} \cup D_{3} \cup D_{5}$ by using operation $S P^{\prime}$. Finally, if $y \notin R_{H} \cup D_{H}^{\prime}$ then $\left(P_{G} \cup N_{G}\right) \times\{y\}$ is dominated by $D_{6}$ because $D_{G}^{\prime}$ dominates $P_{G} \cup N_{G}$.

This proves that $D$ is a dominating set of $G \square H$. To see that $D$ is minimal dominating set we will use Theorem 1. Namely, for each vertex of $D$ we will show that one of the two conditions (i) or (ii) from that theorem holds.

Let $(x, y) \in D_{1}$. If $y \in V(H)$ belongs to $D_{H}^{\prime \prime}$ then clearly $(x, y)$ is not adjacent to any vertex of $D$. If $y \in V(H)$ is from $D_{H}^{\prime}$ then it has a private neighbor $z \in V(H)$. It is clear that $(x, z)$ is a private neighbor of $(x, y)$ (with respect to $D$ ) and so (ii) holds for $(x, y)$.

Let $(x, y) \in D_{2}$. Recall that $D_{2}$ is a maximum independent set of the subgraph induced by $R_{G} \times R_{H}$. And so by definition of independence no two vertices of $D_{2}(=I)$ are adjacent. Other vertices of $D$ that belong to $\{x\} \times V(H)$ or $V(G) \times\{y\}$ also cannot be adjacent to $(x, y)$ since they are obtained by operation $S P$ and belong to $\{x\} \times D_{H}^{\prime}$ and $D_{G}^{\prime} \times\{y\}$, respectively. Recall that $D^{\prime}$ does not have adjacencies with $R$, hence every vertex of $D_{2}$ enjoys condition (i) of Theorem 1 .

If $(x, y) \in D_{3}$, then $y \in S P\left(D_{H}^{\prime}, p_{H}\left(I_{x}\right)\right)$ which means that $y$ enjoys condition $\left(^{*}\right)$ : there exists a vertex $v \in P_{H} \cup N_{H}$ such that $y$ is the only neighbor of $v$ from $S P\left(D_{H}^{\prime}, p_{H}\left(I_{x}\right)\right) \cup I_{x}$. Hence $(x, y)$ is the only neighbor of $(x, v)$ from $D \cap(\{x\} \times V(H))$. It is also clear that $(x, v)$ does not have neighbors in $D \cap(V(G) \times\{v\})$ which implies that $(x, y)$ enjoys condition (ii) of Theorem 1 with respect to $D$.

The case $(x, y) \in D_{4}$ is analog of the previous case and we treat it similarly, concluding that $(x, y)$ enjoys condition (ii) of Theorem 1 .

The case $(x, y) \in D_{5}$ is only slightly different, since the vertex was derived by operation $S P^{\prime}$ on $V(G)$. The minimality condition again implies that there is a vertex $(u, y) \in$ $\left(P_{G} \cup N_{G}\right) \times D_{H}^{\prime}$ such that $(x, y)$ is its only neighbor in $D \cap(V(G) \times\{y\})$. Since there are no vertices in $D \cap\left(\left(P_{G} \cup N_{G}\right) \times V(H)\right)$ we infer that $(u, y)$ is a private neighbor of $(x, y)$ with respect to $D$.

Let $(x, y) \in D_{6}$, that is $x \in D_{G}^{\prime}$ and $y \in P_{H} \cup D_{H}^{\prime \prime} \cup N_{H}$. Note that $x \in V(G)$ has a private neighbor $u \in P_{G}$, and it is clear that $(u, y)$ is a private neighbor of $(x, y)$.

The bound of the theorem is sharp, for instance consider nontrivial paths on at most 3 vertices. It would be interesting to characterize graphs for which the equality is achieved.

We conclude with the following question: can the bound be strengthened to

$$
\Gamma(G \square H) \geq \Gamma(G) \Gamma(H)+\min \{|V(G)|-\Gamma(G),|V(H)|-\Gamma(H)\}
$$

for any nontrivial graphs $G$ and $H$ ? 


\section{References}

[1] W. E. Clark, and S. Suen, An inequality related to Vizing's conjecture, Electron. J. Combin. 7 (2000), no. 1, Note 4, 3 pp. (electronic).

[2] B. Hartnell and D. F. Rall, Domination in Cartesian products: Vizing's conjecture (In: T.W. Haynes, S.T. Hedetniemi and P.J. Slater, Editors, Domination in Graphs Advanced Topics, Marcel Dekker, New York (1998)), 163-189.

[3] T. W. Haynes, S. T. Hedetniemi, and P. J. Slater (eds). Fundamentals of Domination in Graphs, Marcel Dekker, Inc. New York, 1998.

[4] W. Imrich and S. Klavžar, Product Graphs: Structure and Recognition, John Wiley \& Sons, New York, 2000.

[5] R. J. Nowakowski and D. F. Rall, Associative graph products and their independence, domination and coloring numbers, Discuss. Math. Graph Theory 16 (1996), 53-79.

[6] L. Sun, A result on Vizing's conjecture, Discrete Math. 275 (2004), 363-366.

[7] V. G. Vizing, Some unsolved problems in graph theory, Uspehi Mat. Nauk 23 (1968) no. 6 (144), 117-134. 